\title{
Necrotizing Vasculitis
}

National Cancer Institute

\section{Source}

National Cancer Institute. Necrotizing Vasculitis. NCI Thesaurus. Code C70635.

A rare condition that involves inflammation and necrosis of the walls of the blood vessels;

may occur in rheumatoid arthritis. 